University of Nebraska - Lincoln

DigitalCommons@University of Nebraska - Lincoln

Faculty Publications from Nebraska Center for

Materials and Nanoscience, Nebraska Center Materials and Nanoscience

for (NCMN)

August 2003

\title{
Atomic force acoustic microscopy methods to determine thin-film elastic properties
}

\author{
D.C. Hurley \\ National Institute of Standards \& Technology, Boulder, Colorado \\ K. Shen \\ University of Nebraska - Lincoln
}

N.M. Jennett

Materials Centre, National Physical Laboratory, Teddington, Middlesex TW11 OLW, United Kingdom

Joseph A. Turner

University of Nebraska - Lincoln, jaturner@unl.edu

Follow this and additional works at: https://digitalcommons.unl.edu/cmrafacpub

Part of the Nanoscience and Nanotechnology Commons

Hurley, D.C.; Shen, K. ; Jennett, N.M.; and Turner, Joseph A., "Atomic force acoustic microscopy methods to determine thin-film elastic properties" (2003). Faculty Publications from Nebraska Center for Materials and Nanoscience. 56.

https://digitalcommons.unl.edu/cmrafacpub/56

This Article is brought to you for free and open access by the Materials and Nanoscience, Nebraska Center for (NCMN) at DigitalCommons@University of Nebraska - Lincoln. It has been accepted for inclusion in Faculty Publications from Nebraska Center for Materials and Nanoscience by an authorized administrator of DigitalCommons@University of Nebraska - Lincoln. 


\title{
Atomic force acoustic microscopy methods to determine thin-film elastic properties
}

\author{
D. C. Hurley ${ }^{a}$ \\ Materials Reliability Division, National Institute of Standards \& Technology, Boulder, Colorado 80305 \\ K. Shen \\ Department of Engineering Mechanics, University of Nebraska, Lincoln, Nebraska 68588 \\ N. M. Jennett \\ Materials Centre, National Physical Laboratory, Teddington, Middlesex TW11 OLW, United Kingdom \\ J. A. Turner \\ Department of Engineering Mechanics, University of Nebraska, Lincoln, Nebraska 68588
}

(Received 8 April 2003; accepted 23 May 2003)

\begin{abstract}
We discuss atomic force acoustic microscopy (AFAM) methods to determine quantitative values for the elastic properties of thin films. The AFAM approach measures the frequencies of an AFM cantilever's first two flexural resonances while in contact with a material. The indentation modulus $M$ of an unknown or test material can be obtained by comparing the resonant spectrum of the test material to that of a reference material. We examined a niobium film $(d=280 \pm 30 \mathrm{~nm})$ with AFAM using two separate reference materials and two different cantilever geometries. Data were analyzed by two methods: an analytical model based on conventional beam dynamics, and a finite element method that accommodated variable cantilever cross section and viscous damping. AFAM values of $M$ varied significantly depending on the specific experimental configuration and analysis technique. By averaging values obtained with both reference materials, very good agreement $(5-10 \%$ difference) with values determined by other methods was achieved. These results provide insight into using AFAM methods to attain reliable, accurate measurements of elastic properties on the nanoscale. (ㄷ) 2003 American Institute of Physics. [DOI: 10.1063/1.1592632]
\end{abstract}

\section{INTRODUCTION}

Ever-decreasing length scales in many fields of science and technology present a serious challenge for materials characterization. Improved nondestructive measurement tools must be developed to accommodate submicrometer dimensions. Specifically, the ability to determine mechanical properties at the nanoscale is needed, especially for systems involving thin films. Knowledge of mechanical properties such as elastic modulus and interfacial quality (defects, strain, adhesion, etc.) is critical to the successful development of next-generation film materials and structures.

To meet these needs, measurement tools are being developed that exploit the spatial resolution of atomic force microscopy (AFM). Although standard AFM measures topography, other emerging techniques sense a sample's elastic properties. One promising approach is a hybrid acoustic/ AFM technique called atomic force acoustic microscopy (AFAM). ${ }^{1}$ AFAM involves vibrating the cantilever at ultrasonic frequencies to excite its mechanical resonances. The resonant frequencies of the cantilever shift when its tip is brought into contact with a sample. By measuring the resonant frequencies under both free-space and surface-coupled conditions, information about the sample's elastic properties can be extracted. A major advantage of AFAM [and related methods such as ultrasonic force microscopy ${ }^{2}$ (UFM)] is that

\footnotetext{
a)Electronic address: hurley@ boulder.nist.gov
}

the small tip diameter $(\sim 10-100 \mathrm{~nm})$ enables in situ elasticproperty information with nanoscale spatial resolution. Furthermore, AFM's scanning ability means that twodimensional images of mechanical properties are possible.

Although the fundamental principles of AFAM have been established, many aspects of the measurement method are still being refined. We wish to understand which of these aspects most strongly affect AFAM's ability to make quantitative elastic-property measurements. In this paper, we discuss our AFAM measurement and analysis techniques to determine the quantitative elastic properties of thin films. We describe experiments on a thin-film sample using two AFM cantilevers with very different geometries. To understand the results more thoroughly, we compare two methods of AFAM data analysis. Both an analytical approach and a finite element method are used to model the dynamics of the measurement. The same data are interpreted with the two approaches to better understand measurement uncertainty and accuracy. We also compare the AFAM results to those obtained by other techniques such as instrumented indentation ${ }^{3}$ and surface acoustic waves. ${ }^{4}$ In this way, we hope to contribute to the current understanding of quantitative AFAM.

\section{EXPERIMENTAL METHODS}

\section{A. AFAM techniques}

Our experimental apparatus, shown schematically in Fig. 1 , is similar to that of Rabe et al. ${ }^{1}$ The sample under inves- 


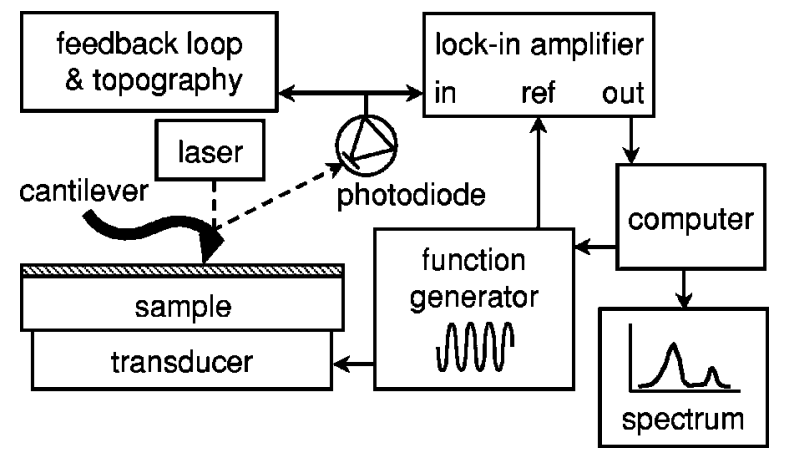

FIG. 1. Schematic of experimental AFAM apparatus.

tigation is bonded to a longitudinal ultrasonic contact transducer that is subsequently affixed to the positioning stage of the AFM instrument. A function generator drives the transducer with a continuous sine wave. The frequency and amplitude of the sine wave (typically $0.1-2.5 \mathrm{MHz}$ and 25-200 $\mathrm{mV}$ ) are computer controlled. When the AFM cantilever tip is in contact with the sample, the transducer vibrations excite resonances in the cantilever through the tip-sample coupling. The response of the AFM photodiode detector then corresponds to the vibration of the cantilever at the transducer frequency. This is detected via a lock-in amplifier whose reference signal is the signal from the function generator. The output signal of the lock-in amplifier is the response of the AFM photodiode detector at the transducer excitation frequency. (At over $3 \mathrm{MHz}$, the photodiode rolloff frequency is higher than the range of frequencies measured.) In a standard experiment, the computer sweeps through a range of excitation frequencies and acquires a spectrum of the cantilever's vibration response versus frequency. Experimental values of the cantilever's first two flexural resonances are determined from this spectrum.

The entire measurement procedure is as follows. First, the free-space resonances of the cantilever are measured by sweeping the transducer frequency while the cantilever is close to, but not touching, the sample (separation $\sim 0.5 \mathrm{~mm}$ ). The transducer vibrations are sufficiently transmitted through the air to excite the cantilever resonances. As discussed below, knowledge of the free-space resonances is needed to characterize the properties of the specific cantilever in use. The cantilever is then lowered and its tip is brought into contact with a reference or calibration sample. Resonant spectra are acquired for one or more values of the force $F_{N}$ applied to the cantilever. $F_{N}$ is related to the cantilever displacement $\delta$ by $F_{N}=k_{c} \delta$, where $k_{c}$ is the spring constant of the cantilever. Thus it is equivalent to obtain spectra for different values of $\delta$, which is simpler to determine experimentally. Typically, measurements are made at three different values in the range $\delta \approx 10-50 \mathrm{~nm}$. For the cantilevers used in these experiments, this corresponds to $F_{N} \approx 0.2-2.2 \mu \mathrm{N}$. Next, the cantilever is brought into contact with the test (unknown) material. The resonances are measured for the same values of $F_{N}$ or $\delta$. The final step in the experimental process is to repeat the measurements on the reference sample. Thus a "data set" usually consists of nine individual measurements of the spectrum: three on the unknown sample and six (a)

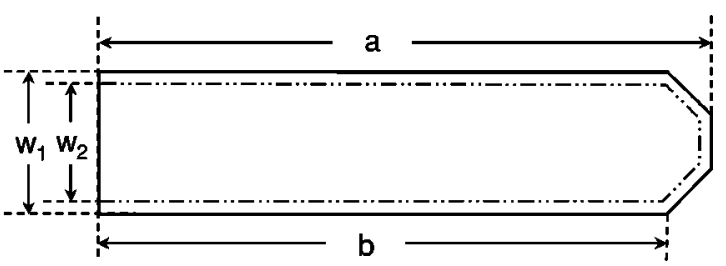

(b)

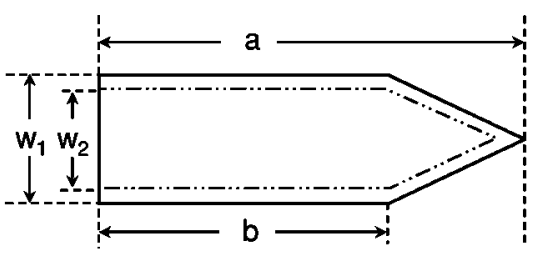

(c)

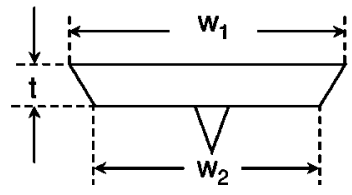

FIG. 2. Geometry of AFM cantilevers used for AFAM experiments. The top two drawings correspond to the plan view of (a) the rectangular cantilever and (b) the dagger cantilever. The cross-sectional diagram in (c) applies to both cantilevers. The drawings in (a) and (b) are approximately to scale.

on the reference material. Here, two reference samples were used and the following sequence of measurements was used: reference 1 , reference 2 , test, reference 2 , reference 1 .

From the experimental resonant frequencies, values are calculated for the tip-sample contact stiffness $k^{*}$ for both the test and reference materials. Further details are given in Sec. III. The calculation is based on a model for the cantilever dynamics. As described later, we have implemented both analytical and numerical approaches to the model. Finally, the values of $k^{*}$ for the test and reference samples are compared in order to obtain the desired quantity, the elastic modulus of the test sample.

It can be seen that this measurement procedure relies on one or more reference samples with known elastic properties. Comparison of test and reference data eliminates the need for precise knowledge of parameters that are difficult to determine experimentally, for instance the tip radius. ${ }^{5}$ The procedure yields two sets of results: one for the comparison between the test data and the first set of reference data, and one between the test data and the second set of reference data. The two sets of results are typically averaged to obtain a single value of the modulus. In this way, effects such as tip wear can be minimized.

\section{B. Cantilever types}

Measurements were made with two different cantilevers with different geometries. Diagrams of the cross section and plan view for both cantilevers are shown in Fig. 2. The dimensions of the cantilevers and their first two free-space flexural resonances are given in Table I. Dimensions were determined with an optical microscope, except for the thicknesses, which were measured with a scanning electron microscope (SEM).

The plan view of the first cantilever is shown in Fig. 2(a). It was nearly rectangular and thus was referred to as the 
TABLE I. Properties of cantilevers used in AFAM experiments. Entries include the dimensions defined in Fig. 2 and the frequencies of the two lowest free-space flexural resonances $f_{1}^{0}$ and $f_{2}^{0}$. The columns labeled "actual" indicate the actual measured values. Columns labeled "FEM" contain the values used in, or obtained by, the finite element method.

\begin{tabular}{lcccc}
\hline \hline & \multicolumn{4}{c}{ Cantilever type } \\
\cline { 2 - 5 } Property & Actual & FEM & Actual & FEM \\
\cline { 2 - 5 } & $232 \pm 2$ & 232.0 & $156 \pm 1$ & 156.9 \\
$a(\mu \mathrm{m})$ & $223 \pm 2$ & 223.0 & $101 \pm 1$ & 100.8 \\
$b(\mu \mathrm{m})$ & $52 \pm 1$ & 52.0 & $49 \pm 1$ & 48.0 \\
$w_{1}(\mu \mathrm{m})$ & $42 \pm 1$ & 42.0 & $39 \pm 2$ & 38.7 \\
$w_{2}(\mu \mathrm{m})$ & $8.1 \pm 0.2$ & 7.72 & $4.1 \pm 0.2$ & 3.85 \\
$t(\mu \mathrm{m})$ & $180.8 \pm 0.2$ & 180.8 & $257.9 \pm 0.2$ & 257.9 \\
$f_{1}^{0}(\mathrm{kHz})$ & $1157.8 \pm 0.2$ & 1158.0 & $1427.7 \pm 0.2$ & 1427.7 \\
$f_{2}^{0}(\mathrm{kHz})$ & \multicolumn{4}{c}{} \\
\hline \hline
\end{tabular}

"rectangular" cantilever. The cantilever had a uniform, trapezoidal cross section along its length except at the very end. The tip was located near, but not exactly at, the end of the cantilever. The position of the tip $L_{1}$ relative to the total length $L$ of the cantilever can be characterized by the ratio $L_{1} / L{ }^{1}$ As discussed below, $L_{1} / L$ is used as an adjustable parameter in the data analysis. By examining the cantilever in the SEM and the optical microscope, we estimated that $L_{1} / L=0.95-0.97$.

The second cantilever did not possess a rectangular geometry. Due to its shape, seen in Fig. 2(b), it was called the "dagger" cantilever. The figure indicates that the cantilever's cross section was not uniform across its entire length. Although trapezoidal throughout, the cross section varied in width along the pointed (triangular) region. Values for the widths $w_{1}$ and $w_{2}$ given in Table I correspond to the cantilever's rectangular region furthest from the tip. This type of cantilever is attractive for AFAM experiments, partly because the tip is located at exactly the end of the cantilever so that $L_{1} / L=1.0$. In addition, the angle between the tip and the cantilever is tilted by approximately $12^{\circ}$ to compensate for the angle at which the cantilever is mounted in most commercial AFM instruments. Therefore, when this tip is brought into contact with a sample, it is perpendicular to the sample surface. We have found this arrangement to work well in practice.

The two cantilevers varied not only in their geometry, but also in the relative values of the cantilever spring constant $k_{c}$. For our particular rectangular cantilever, a value of $k_{c}=45.2 \mathrm{~N} / \mathrm{m}$ was provided by the vendor. The precise value of $k_{c}$ was not given for the dagger cantilever, but a range of possible values $k_{c} \approx 29-55 \mathrm{~N} / \mathrm{m}$ was specified. The relative values of $k_{c}$ for the two cantilevers can be estimated using the relation $k_{c}=E w t^{3} / 4 L^{3}$ for a rectangular beam. Here, $E$ is Young's modulus, $w$ is the width, $t$ is the thickness, and $L$ is the length of the cantilever. Inserting the appropriate values in Table I into this equation, we find that the rectangular cantilever is about 2.5 times stiffer than the dagger cantilever. Because the equation applies to a rectangular beam, this is only an approximation. However, the ratio is likely to be even greater since the taper in the dagger cantilever makes it less stiff than a rectangular beam of the same length and thickness.

\section{Sample materials}

To test the methods described above, we performed AFAM experiments on a thin film of niobium $(\mathrm{Nb})$. The film was sputtered onto a (001) single-crystal silicon ( $\mathrm{Si}$ ) wafer approximately $0.5 \mathrm{~mm}$ thick. The specific film and substrate materials were chosen based on expected values of the elastic properties, ease of fabrication, and availability of literature values for comparison. The film thickness $d_{\mathrm{Nb}}$ was measured by breaking the sample and examining it in cross section in the SEM. A value $d_{\mathrm{Nb}}=280 \pm 30 \mathrm{~nm}$ was obtained by averaging a total of 26 measurements acquired at seven evenly spaced positions over a distance of $22 \mathrm{~mm}$. The uncertainty in the thickness represents the standard deviation of the individual measurements. AFM topography measurements of the surface roughness indicate that the rms roughness of the $\mathrm{Nb}$ film was $1.5-2.0 \mathrm{~nm}$.

Quantitative AFAM measurements require in situ calibration with a reference sample. The elastic properties of the reference sample, namely, its indentation modulus $M$ $=E /\left(1-\nu^{2}\right)$, where $E$ is Young's modulus and $\nu$ is Poisson's ratio, must be known. In previous research, a reference material with properties close to those expected in the test material has usually been selected. From literature values for bulk $\mathrm{Nb}$, we estimate that $M_{\mathrm{Nb}}=116-133 \mathrm{GPa} .{ }^{6}$

We could not identify an easily available reference material with $M$ in this range. Instead, we used two calibration samples whose properties bracketed these values. The first was a (001) single-crystal Si wafer approximately $0.5 \mathrm{~mm}$ thick. We expect $M_{\mathrm{Si}\langle 100\rangle}=161 \mathrm{GPa}$ from calculations of the effective anisotropic values for $E_{\mathrm{Si}\langle 100\rangle}$ and $\nu_{\mathrm{Si}\langle 100\rangle}$ from the second-order elastic moduli of silicon. ${ }^{7-9}$ However, a value of $M_{\mathrm{Si}}=139 \pm 10 \mathrm{GPa}$ was obtained with instrumented indentation testing (IIT) on the specific sample used. The IIT measurements also indicated that $M$ was slightly depth dependent, increasing to about $170 \mathrm{GPa}$ at deeper penetration depths-closer to the expected value. It is possible that the sample possessed a thin surface layer of native oxide or polishing damage. Because the applied AFAM forces were smaller than those used in the IIT, we used the surface value $M_{\mathrm{Si}}=139 \mathrm{GPa}$ in the AFAM data analysis.

The other reference sample was a disk of borosilicate crown glass. Its properties were characterized using standard immersion, pulse-echo ultrasonic techniques. ${ }^{10}$ Measurements of the longitudinal and shear wave velocities in the glass yielded an indentation modulus $M_{g l}=85 \pm 3 \mathrm{GPa}$, identical to the nominal value quoted by the vendor.

\section{DATA ANALYSIS METHODS}

\section{A. Analytical method}

The standard approach for interpreting AFAM frequency data uses conventional beam dynamics to obtain an analytical relation between the contact stiffness and the resonant frequencies. This approach has been described in detail elsewhere; 1,5 here, we only summarize the basic concepts. The drawing in Fig. 3 depicts the key features of the model. 


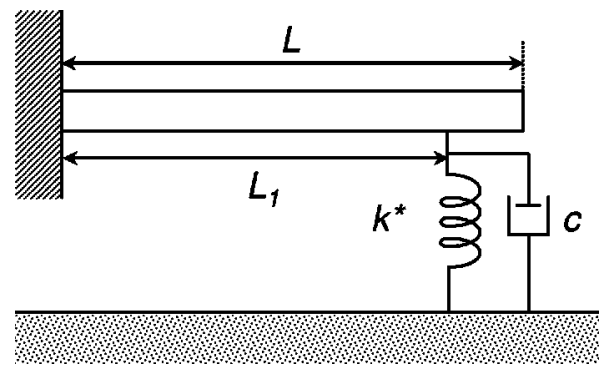

FIG. 3. Diagram of key features of AFAM model. The cantilever is clamped at one end and has a total length $L$. It is coupled to the surface through a spring of stiffness $k^{*}$ (contact stiffness) located at a position $L_{1}$ with respect to the clamped end. An optional dashpot with damping constant $c$ is located in parallel with the spring.

The cantilever is modeled as a beam of length $L$ with a perfectly uniform cross section. One end of the cantilever is clamped. The other (tip) end is free to vibrate (free-space condition) or else coupled to the surface by a spring of stiffness $k^{*}$ (sample-coupled condition). The spring is located at position $L_{1}$ with respect to the clamped end of the cantilever.

Closed-form analytical expressions can be written to characterize the beam dynamics of this system. The equations relate the frequencies of the first two free-space flexural resonances $f_{1}^{0}$ and $f_{2}^{0}$ to a characteristic parameter $c_{B} \cdot c_{B}$ contains the cantilever mass density, Young's modulus, and beam thickness. ${ }^{1}$ The parameter $c_{B}$ is combined with the sample-coupled resonant frequencies to form the argument of a characteristic equation for the sample-coupled vibrations. This transcendental equation is solved to determine the value of the contact stiffness $k^{*}$ between the tip and the sample.

From values of $k^{*}$ and knowledge of the reference material's elastic properties, first the reduced Young's modulus $E^{*}$ and then the indentation modulus $M_{\text {test }}$ can be calculated $^{5}$ :

$$
\begin{aligned}
& E_{\text {test }}^{*}=E_{\text {ref }}^{*}\left(\frac{k_{\text {test }}^{*}}{k_{\text {ref }}^{*}}\right)^{n}, \\
& \frac{1}{E_{\text {test }}^{*}}=\frac{1}{M_{\text {tip }}}+\frac{1}{M_{\text {test }}} .
\end{aligned}
$$

Here, the subscript test indicates the unknown sample and ref refers to the reference or calibration sample. We used a value $M_{\text {tip }}=M_{\mathrm{Si}\langle 001\rangle}=161 \mathrm{GPa}$ for the $\langle 001\rangle$ silicon tip. The value of $n$ in Eq. (1) depends on the contact mechanics model used. For Hertzian contact, $n=3 / 2$; for a flat-punch (flat) contact, $n=1 .{ }^{5}$ We cite the values of $M$ calculated for both $n=1$ and $n=3 / 2$ to indicate the range of possible values. It should be noted that contact mechanics models in which the effects of adhesion are included were not considered.

In theory, the frequency of only one flexural resonance is needed to determine $k^{*}$ with this model. In practice, the frequencies of two or more modes are measured and $k^{*}$ is calculated for each one. This practice is partially motivated by the fact that depending on the experimental configuration, one mode is usually more sensitive to changes in $k^{*}$. ${ }^{11}$ The values for $k^{*}$ obtained for different resonances, however, do not exactly agree if the assumed position of the AFM tip is the very end of the cantilever (that is, if $L_{1} / L=1.0$ ). Therefore, the characteristic equation for sample-coupled vibration has been modified to account for the possibility $L_{1} / L<1.0{ }^{1}$ In this case, $k^{*}$ is plotted as a function of $L_{1} / L$ for each flexural mode. The value of $k^{*}$ where the curves intersect is considered the solution for $k^{*}$. Thus $L_{1} / L$ can be considered an adjustable parameter in the analytical method. Typically, $L_{1} / L=0.91-0.99$ depending on the specific cantilever geometry and other experimental variables. This procedure is usually carried out for each pair of resonant frequencies $\left(f_{1}, f_{2}\right)$ separately.

Strictly speaking, the analytical model should not be applied to the dagger cantilever because its geometry does not meet the assumptions of the model. However, this model has been shown to be an effective means of analyzing AFAM experimental data and is in fact the only method widely available. Therefore, we will include results obtained by the analytical method for comparison to results with the numerical (finite element) method described below.

\section{B. Finite element method}

To date, AFAM experiments have been interpreted exclusively with the above analytical method. This approach is somewhat limited in applicability, since it assumes that the cantilever's cross section is exactly the same along its entire length. The assumption is not strictly true, even for cantilevers like our rectangular one. Thus data interpretation with the analytical model is based on an approximation to the actual experimental conditions. Previous work as well as our own results indicate that the approximation is a very good one in some cases. However, because the measured resonant frequencies for different flexural modes do not predict exactly the same value of $c_{B}$, it is clear that real cantilevers do not exactly fit the analytical model. Furthermore, cantilevers such as our dagger cantilever may be valuable for experimental use but do not satisfy the assumptions of the model.

To address these issues and to explore whether another approach might improve measurement accuracy, we have developed a numerical method for AFAM analysis. Many approaches may be used to create a model to describe the AFM cantilever vibrations. Although it is a likely candidate, an expansion in basis functions that span the length of the cantilever (e.g., Rayleigh-Ritz) is not the most convenient for the cantilevers studied here. Variations in geometry that extend over a limited range of the cantilever, such as the triangular portion of the dagger probe, are more easily modeled using a solution derived by the finite element method (FEM). With the FEM, the geometric and mechanical properties of each element can be varied independently.

A finite element mesh was created for each cantilever type based on the dimensions in Fig. 2. Each cantilever was discretized into Timoshenko beam elements that included the rotational inertia of the element. The number of elements was chosen for good convergence of the first five flexural modes when compared with exact solutions for cantilevers with uniform cross sections. For the rectangular cantilever 107 beam elements were used, while the mesh for the dagger cantilever contained 247 elements. The numerical results 
given here were calculated assuming that all of the elements possessed the same material properties.

The dimensions of the cantilever mesh were initially assigned the measured values in Table I. For the rectangular cantilever, the width, length, and thickness were then adjusted to match the free-space response of the FEM model beam to the experimental results. We were able to match the experimental frequencies by making only small adjustments within the uncertainty of the dimensional measurements. Next, a linear thickness gradient of $5.0 \%$ along the length of the cantilever was introduced to precisely match the ratio of the first two resonant frequencies to the equivalent experimental ratio. The frequency ratio is nearly unaffected by uniform changes in width or thickness and hence is an indicator of the variation in thickness along the cantilever. Examination of the cantilever in the SEM indicated that this amount of thickness variation was reasonable. The same approach was used for the dagger cantilever, except that the length of the dagger point or triangle was also adjusted. In this case, a thickness gradient of only $3.5 \%$ was used.

The values used in the FEM calculations are given in Table I. Also shown are the values of the first two flexural free resonances calculated from the FEM dimensions. The agreement in both dimensions and frequency with the actual (measured) values is very good for both cantilevers. However, it should be noted that our combination of modeling parameters is not a unique solution. It is possible to obtain similar results for the cantilever free frequencies using other combinations of $E_{\mathrm{Si}}$ and $t$. The values in Table I represent one of these combinations that yields values close to the measured ones.

Once the optimum mesh dimensions were determined, the contact vibration response could be calculated. The contact model contained a spring of stiffness $k^{*}$ located at one node of the mesh. The parameter $L_{1} / L$ described the location of the node with the spring. $L_{1} / L$ was allowed to vary between 0.9 and 1.0 for the rectangular cantilever. The FEM calculation then involved predicting values of $f_{1}$ and $f_{2}$ for each combination of $k^{*}$ and $L_{1} / L$. The output values were those values of $k^{*}$ (and $L_{1} / L$ ) that gave the best agreement between the predicted and experimental frequencies. "Best agreement" meant that the values minimized the sum of the error between experimental and numerical values for both $f_{1}$ and $f_{2}$. Specifically, we sought to minimize an error function $\chi$, where

$$
\chi=10 \frac{\left|f_{1}-f_{1}^{T}\right|}{f_{1}}+\frac{\left|f_{2}-f_{2}^{T}\right|}{f_{2}} .
$$

The superscript $T$ indicates the numerical value and the unsuperscripted value corresponds to the measured value. The maximum error allowed was $0.3 \%$. An error weighting of 10:1 was used to compensate for the different sensitivities of $f_{1}$ and $f_{2}$ to changes in surface stiffness. ${ }^{11}$ For the relevant range of values of $k^{*}$, the 10:1 ratio causes the magnitude of the errors associated with both $f_{1}$ and $f_{2}$ to be of roughly equal importance. Thus, for some cases, the match for either frequency may not have appeared optimized, but the condition on the minimum error was met. It should also be noted that the search space was limited to local minima. The possibility of finding a global minimum of $\chi$ was not explored.

Our first numerical approach for the dagger cantilever duplicated that used for the rectangular, that is, only $L_{1} / L$ and $k^{*}$ were varied. Although physically realistic values were obtained for $L_{1} / L(0.97-0.99)$, the values of $k^{*}$ for different frequency modes with the same cantilever did not agree with only these parameters. Therefore, a viscous damper was added to the numerical model. This addition was motivated partly because the experimental data indicated a greater effect of damping for the dagger cantilever as evidenced by larger resonant linewidths. Viscous damping was included in the form of a dashpot with damping constant $c$ in parallel with the spring corresponding to $k^{*}$, as shown in Fig. 3. For the minimization procedure, $L_{1} / L$ and $k^{*}$ were first varied to minimize the error. Then, an appropriate value of $c$ was added to further reduce the error. The process was repeated until the required error level was achieved. For this cantilever, the range over which $L_{1} / L$ was allowed to vary was limited to $0.97-1.0$.

The predicted response of the dagger cantilever was quite sensitive to the value of $c$ used in the calculation. To obtain good agreement with the experimental results, the values of $c$ varied by more than a factor of 100 for some of the data sets. The reasons for the variation in $c$ are not clear. Experimentally, we have observed that the damping can be a function of the applied load. We have also found that the importance of damping was dependent on the cantilever stiffness. Data from the rectangular cantilever, which was stiffer, were analyzed using the model that included damping. We found that these results were relatively insensitive to the value of $c$. For the less stiff dagger cantilever, we could not match the numerical and experimental results unless damping was included. The relevant levels of cantilever stiffness that necessitate the use of damping have not yet been determined. However, the influence of contact damping level on AFM vibrations has been discussed previously. ${ }^{12}$ More sophisticated models of the tip damping are currently under development.

The procedures described above were performed for each separate AFAM measurement. Each calculation for the optimum contact parameters required less than five minutes on a desktop computer (processing speed $1 \mathrm{GHz}$ ). The first calculation for each material/cantilever combination was the most time consuming. Subsequent searches using the first results as an initial guess required less time. Once the values for $k^{*}$ were determined, the analysis procedure was the same as for the analytical method: the ratio $k_{\text {test }}^{*} / k_{\text {ref }}^{*}$ was calculated and $M_{\text {test }}$ evaluated from Eqs. (1) and (2).

\section{RESULTS AND DISCUSSION}

Table II summarizes our AFAM results for the $\mathrm{Nb}$ film sample. Values are shown for the indentation modulus $M_{\mathrm{Nb}}$ as determined using different combinations of cantilever geometry, reference material, and analysis approach. Values of $M_{\mathrm{Nb}}$ are shown for both $n=1$ and $n=3 / 2$ in Eq. (1). Each entry in Table II represents the average of four data sets; as described in Sec. II A, each data set typically yielded six 
TABLE II. AFAM values for the indentation modulus $M_{\mathrm{Nb}}$ of $\mathrm{Nb}$ film. The type of cantilever, reference material, and analysis model used to determine $M_{\mathrm{Nb}}$ are indicated. Values for $M_{\mathrm{Nb}}$ were determined from Eqs. (1) and (2) using $n=1$ or $n=3 / 2$, as indicated.

\begin{tabular}{|c|c|c|c|c|}
\hline Cantilever & Reference & Model & $\begin{array}{c}M(n=1) \\
\quad(\mathrm{GPa})\end{array}$ & $\begin{array}{c}M(n=3 / 2) \\
(\mathrm{GPa})\end{array}$ \\
\hline \multirow[t]{6}{*}{ Rectangular } & \multirow[t]{2}{*}{ Glass } & Analytical & $88 \pm 9$ & $90 \pm 14$ \\
\hline & & FEM & $89 \pm 11$ & $92 \pm 17$ \\
\hline & \multirow[t]{2}{*}{$\mathrm{Si}$} & Analytical & $127 \pm 7$ & $122 \pm 10$ \\
\hline & & FEM & $126 \pm 8$ & $120 \pm 12$ \\
\hline & \multirow[t]{2}{*}{ Average } & Analytical & $106 \pm 12$ & $105 \pm 18$ \\
\hline & & FEM & $106 \pm 14$ & $105 \pm 22$ \\
\hline \multirow[t]{6}{*}{ Dagger } & \multirow[t]{2}{*}{ Glass } & Analytical & $86 \pm 2$ & $87 \pm 3$ \\
\hline & & FEM & $86 \pm 3$ & $87 \pm 4$ \\
\hline & \multirow[t]{2}{*}{$\mathrm{Si}$} & Analytical & $127 \pm 5$ & $121 \pm 7$ \\
\hline & & FEM & $118 \pm 4$ & $110 \pm 5$ \\
\hline & \multirow[t]{2}{*}{ Average } & Analytical & $105 \pm 5$ & $103 \pm 7$ \\
\hline & & FEM & $101 \pm 5$ & $98 \pm 6$ \\
\hline
\end{tabular}

values of $M_{\mathrm{Nb}}$. The uncertainties quoted in Table II represent the standard deviation of these multiple measurements. The accuracy of the value used for $M_{r e f}$ is potentially an additional source of measurement uncertainty. If the true value of $M_{r e f}$ differs from the value used, a systematic measurement error exists. The value assumed for $M_{\text {tip }}$ can also systematically change the measurement results. However, this effect is quite small. We found that changes in $M_{t i p}$ of $20-25 \mathrm{GPa}$ changed the calculated values of $M_{\mathrm{Nb}}$ by less than $1 \mathrm{GPa}$.

Table II contains additional entries labeled "average." These indicate the values of $M_{\mathrm{Nb}}$ obtained by calculating an average $E^{*}$ from all measurements with both reference materials and then computing $M$ with Eq. (2). Due to the relationship between $E^{*}$ and $M$, this value differs slightly from the average of the two final values of $M_{\mathrm{Nb}}$ obtained with the separate reference materials. The uncertainty for the averages was calculated from an uncertainty in reduced modulus $\delta E_{\text {avg }}^{*}$ determined by $\delta E_{\text {avg }}^{*}=\sqrt{\left(\delta E_{g l}^{*}\right)^{2}+\left(\delta E_{\mathrm{Si}}^{*}\right)^{2}}$, where $\delta E_{g l}^{*}$ and $\delta E_{\mathrm{Si}}^{*}$ are the standard deviations in the measurements from each separate reference material.

For comparison with the AFAM results, Table III contains values for $M_{\mathrm{Nb}}$ obtained by other methods. The first column in Table III indicates a range of values for polycrystalline bulk $\mathrm{Nb}$ obtained from the literature. ${ }^{6}$ The second and third columns contain values for $M_{\mathrm{Nb}}$ measured on the same $\mathrm{Nb}$ film sample with surface acoustic wave spectroscopy ${ }^{4,13}$ (SAWS) and instrumented indentation testing. ${ }^{3}$ The values indicated are the average of multiple measurements on the same sample. In the analysis of the SAWS data, the film density $\rho$ was assumed to be that of bulk $\mathrm{Nb}$ ( $\rho$ $=8570 \mathrm{~kg} / \mathrm{m}^{3}$ ). Both $\rho$ and $M$ could not be determined si-

TABLE III. Values for the indentation modulus $M$ of $\mathrm{Nb}$ (in GPa). A range of values for bulk $\mathrm{Nb}$ obtained from the literature is shown. Also included are results obtained by surface acoustic wave spectroscopy (SAWS) and instrumented indentation testing (IIT) on the same sample measured by AFAM. The minimum and maximum AFAM values from Table II are given.

\begin{tabular}{lccc}
\hline \hline Literature (bulk) & SAWS & IIT & AFAM \\
\hline $116-133$ & $121 \pm 7$ & $97 \pm 10$ & $86-127$ \\
\hline \hline
\end{tabular}

multaneously from the SAWS data. If the actual film density was lower, as can be the case for thin films, $M_{\mathrm{Nb}}$ would be lower than that shown. To obtain the IIT value of $M_{\mathrm{Nb}}$ $=97 \mathrm{GPa}$ from our SAWS data, the film density would have to be $\sim 5 \%$ less than the bulk value.

Inspection of Tables II and III provides insight into the accuracy and effectiveness of our methods. For the rectangular cantilever, the analysis approach had virtually no effect on the resulting value of $M_{\mathrm{Nb}}$. Discrepancies in the value of $M_{\mathrm{Nb}}$ between the two models are much smaller than the measurement uncertainty for both reference materials. Thus the results from the two models can be considered identical within the uncertainty. For the dagger cantilever, results with the glass reference sample are identical for both analysis approaches. Using the Si reference sample, however, results with the analytical method are 8-10\% higher than those obtained by FEM analysis of the same data. Although the discrepancies between the two models were larger than the measurement uncertainty, the error bars still overlap and thus the differences are not considered significant. Thus we conclude that our finite element models successfully captured the key physical features of the systems.

Tables II and III indicate that better agreement between the AFAM and IIT results is achieved by averaging AFAM results from two reference materials. For the rectangular cantilever, $M_{\mathrm{Nb}}$ determined by the Si reference sample is higher than $M_{\mathrm{Nb}}$ (IIT) by $20-30 \%$, while $M_{\mathrm{Nb}}$ determined by the glass reference sample alone is lower by $\sim 10 \%$. When the two data sets are combined, $M_{\mathrm{Nb}}(\mathrm{AFAM})=105-106 \mathrm{GPa}$ is obtained. This is in very good agreement with the IIT value (higher by $\sim 5 \%$ and within measurement uncertainty). For the dagger cantilever, $M_{\mathrm{Nb}}$ for the average of the two reference materials from the analytical model is slightly higher than the corresponding FEM results. However, the values from the two models are the same within measurement uncertainty. The results are also identical within the error bars to the corresponding values with the rectangular cantilever. In fact, the average values of $M_{\mathrm{Nb}}$ for the dagger cantilever agree more closely with the IIT values. The consistency between AFAM results from different cantilever geometries and analysis approaches, as well as the good agreement with results from other methods, strongly suggests that our AFAM methods are valid.

In these AFAM experiments two different reference materials were used for quantitative measurements. Prasad et al. ${ }^{14}$ qualitatively compared the AFAM spectrum of a test material (dickite) to those of other materials in order to select a single reference material. Our results suggest that with the current analysis approach, the choice of reference material is critical to measurement accuracy. It appears that if $M_{r e f}$ differs substantially from $M_{\text {test }}$, the experimentally determined $M_{\text {test }}$ may be incorrect. Such behavior might occur if the contact between the nonideal AFM tip and the nonideal surface differs for different sample materials. The Hertzian contact mechanics model used for our data analysis does not include such variability. Until this can be verified and a more detailed contact mechanics model developed, our results indicate that reasonable values of $M_{\text {test }}$ can be obtained using two reference materials. Multiple reference samples may be 
needed if no single suitable reference material is available, as was the case here. More importantly, an estimate of $M_{\text {test }}$ may not be available, as can occur in the development of film materials. In this case, AFAM measurements with multiple references could serve to iteratively determine the value of $M_{\text {test }}$. As the AFAM technique is refined and its precision is increased, we hope to examine this behavior more thoroughly and determine how measurement accuracy can be improved.

A question that arises in this context is how similar $M_{\text {test }}$ and $M_{\text {ref }}$ should be to obtain accurate results. To address this question, we compare these experiments with previous ones we have performed. ${ }^{15}$ With AFAM we measured an aluminum (Al) film about $1 \mu \mathrm{m}$ thick. In this case, only the glass sample was used as a reference $\left(M_{g l}=85 \pm 3 \mathrm{GPa}\right)$. AFAM data obtained with the rectangular cantilever and analyzed with the analytical model yielded $M_{\mathrm{Al}}(\mathrm{AFAM})=80 \pm 3 \mathrm{GPa}$ $(n=3 / 2)$. The SAWS value for the same sample was $M_{\mathrm{Al}}(\mathrm{SAWS})=78 \pm 1 \mathrm{GPa}$. Thus AFAM results with a single reference appeared accurate when $M_{\text {test }}$ differed from $M_{\text {ref }}$ by $5-10 \%$. Assuming that the true value of $M_{\mathrm{Nb}}$ is approximately $100 \mathrm{GPa}$, then for the current experiments $M_{\mathrm{Si}}$ was about $40 \%$ higher and $M_{g l}$ was $15-20 \%$ lower than $M_{\mathrm{Nb}}$. Further experiments are needed to more fully quantify this behavior.

As mentioned above, both analysis methods used a variable $L_{1} / L$ describing the tip position as an adjustable parameter. The value of $L_{1} / L$ was determined for each separate $\left(f_{1}, f_{2}\right)$ data pair. For the rectangular cantilever, the range of values obtained with the analytical approach was $L_{1} / L$ $=0.913-0.926$ while $L_{1} / L=0.916-0.930$ for the FEM approach. For the dagger cantilever, $L_{1} / L=0.57-0.58$. This is quite different from the value $L_{1} / L=1$ observed in the SEM and illustrates our interest in developing an alternative analysis approach. With the finite element method, $L_{1} / L$ $=0.975-0.985$ for the dagger cantilever. Further analysis or more sophisticated modeling is needed to clarify the slight but consistent differences in $L_{1} / L$ between the observed and model values.

\section{SUMMARY AND CONCLUSIONS}

We have described AFAM methods to determine the quantitative elastic properties of thin films. Experiments were performed on a metallic thin-film sample using two cantilevers with different geometries. The cantilever resonant frequencies for the lowest two flexural modes were measured both in free space and while the tip was in contact with the sample. Comparison of results to those from reference samples with known elastic properties enabled the sample indentation modulus $M$ to be determined. Data were analyzed with two methods. The first was an analytical model for beam dynamics that assumed a perfectly uniform cantilever cross section. The second involved a finite element method specifically developed to accommodate variations in thickness and width along the length of the cantilever.

With these measurement and analysis methods, we determined AFAM values for $M$ in a niobium film $280 \mathrm{~nm}$ thick. Measurements were made on the same film with two other techniques: surface acoustic wave spectroscopy and instrumented indentation. The agreement between these values and those determined with AFAM was only fair (10-25\% different) if a single reference material was used for the AFAM measurements. Much better agreement ( $\sim 5 \%$ difference) was achieved by combining AFAM measurements from two different reference samples.

For a cantilever geometry that deviated only slightly from a perfect rectangular beam, the two analysis methods yielded values for $M$ that were the same within measurement uncertainty. Thus the analytical model-a much simpler approach-appears adequate for this type of geometry. For cantilevers with a nonuniform cross section, it was necessary to include the effects of viscoelastic damping in the FEM analysis. FEM values for $M_{\mathrm{Nb}}$ with this cantilever were the same within measurement uncertainty as those with the rectangular cantilever. Although not strictly applicable to this cantilever geometry, the rectangular model produced very similar values for $M_{\mathrm{Nb}}$. However, its values of the tip parameter $L_{1} / L$ were unphysical. Therefore we believe that the FEM approach should be applied to cantilevers with distinctly nonrectangular geometry.

These results demonstrate the validity of our AFAM methods for quantitative measurements of the elastic properties of thin films and surfaces. Our work indicates that for optimal results, a reference material should be selected with mechanical properties (modulus, adhesion, etc.) similar to those of the test material. In this way, the experimental contact radii will be similar and the assumptions of the AFAM model will be valid. Future work includes extending the measurement techniques to additional samples with a wider range of $M$ in order to investigate the limits of applicability in more detail. We also plan to examine the issues that affect precision and repeatability more thoroughly. Furthermore, other cantilever geometries will be examined with FEM calculations to explore the utility of the approach.

\section{ACKNOWLEDGMENTS}

This work was sponsored in part (J.A.T. and K.S.) by the Air Force Office of Scientific Research (Grant No. F4962099-1-0254) and the National Science Foundation (Grant No. DMI-0210850). We thank P. D. Dresselhaus (NIST) for fabricating the $\mathrm{Nb}$ sample, $\mathrm{P}$. S. Rice (NIST) for assistance with AFAM measurements, and G. Aldrich-Smith (NPL) for assistance with IIT and AFM measurements. We are grateful to U. Rabe, W. Arnold, M. Kopycinska, and S. Hirsekorn (IZFP, Germany) for many valuable technical discussions.

${ }^{1}$ U. Rabe, S. Amelio, E. Kester, V. Scherer, S. Hirsekorn, and W. Arnold, Ultrasonics 38, 430 (2000).

${ }^{2}$ K. Yamanaka, A. Noguchi, T. Tsuji, T. Koike, and T. Goto, Surf. Interface Anal. 27, 600 (1999).

${ }^{3}$ W. C. Oliver and G. M. Pharr, J. Mater. Res. 7, 1564 (1992).

${ }^{4}$ A. Lomonosov, A. P. Mayer, and P. Hess, in Handbook of Elastic Properties of Solids, Liquids, and Gases, edited by M. Levy, H. Bass, and R. Stern (Academic Press, San Diego, 2001), Vol. 1, Chap. 7, pp. 137-186.

${ }^{5}$ U. Rabe, S. Amelio, M. Kopycinska, S. Hirsekorn, M. Kempf, M. Göken, and W. Arnold, Surf. Interface Anal. 33, 65 (2002).

${ }^{6}$ G. Simmons and H. Wang, Single Crystal Elastic Constants and Calculated Aggregated Properties: A Handbook (MIT Press, Cambridge, 1971). 
${ }^{7}$ G. Grimvall, Thermophysical Properties of Materials (Elsevier, New York, 1999).

${ }^{8}$ H. J. McSkimin and P. A. Andreatch, J. Appl. Phys. 35, 2161 (1964).

${ }^{9}$ J. J. Vlassak and W. D. Nix, Philos. Mag. A 67, 1045 (1993).

${ }^{10}$ E. P. Papadakis, in Physical Acoustics, Vol. 12, edited by W. P. Mason and

R. N. Thurston (Academic Press, San Diego, 1976), p. 277.

${ }^{11}$ J. A. Turner and J. S. Wiehn, Nanotechnology 12, 322 (2001).

${ }^{12}$ J. A. Turner, S. Hirsekorn, U. Rabe, and W. Arnold, J. Appl. Phys. 82, 966
(1997)

${ }^{13}$ D. C. Hurley, V. K. Tewary, and A. J. Richards, Meas. Sci. Technol. 12, 1486 (2001).

${ }^{14}$ M. Prasad, M. Kopycinska, U. Rabe, and W. Arnold, Geophys. Res. Lett. 29, 10.1029/2001/GL014054 (2002).

${ }^{15}$ D. C. Hurley, J. S. Wiehn, J. A. Turner, and P. S. Rice, in SPIE Symposium Proceedings, Vol. 4703, edited by N. Meyendorf, G. Y. Baaklini, and B. Michel (SPIE, Bellingham, WA, 2002), p. 65. 